\title{
Left Ventricular Reconstruction and Mitral Valve Annuloplasty Combined with Papillary Muscle Relocation for Severe Ischemic Mitral Regurgitation
}

\author{
Koji Furukawa1,2*, Mitsuhiro Yano1, Eisaku Nakamura², Masakazu Matsuyama1, \\ Masanori Nishimura ${ }^{1}$, Katsuya Kawagoe ${ }^{1}$, Kunihide Nakamura ${ }^{2}$ \\ ${ }^{1}$ Department of Cardiovascular Surgery, Miyazaki Medical Association Hospital, Miyazaki, Japan \\ ${ }^{2}$ Department of Cardiovascular Surgery, Faculty of Medicine, University of Miyazaki, Miyazaki, Japan \\ Email: ${ }^{\star}$ koji_furukawa@med.miyazaki-u.ac.jp
}

How to cite this paper: Furukawa, K., Yano, M., Nakamura, E., Matsuyama, M., Nishimura, M., Kawagoe, K. and Nakamura, K. (2017) Left Ventricular Reconstruction and Mitral Valve Annuloplasty Combined with Papillary Muscle Relocation for Severe Ischemic Mitral Regurgitation. World Journal of Cardiovascular Surgery, 7, 150155.

https://doi.org/10.4236/wjcs.2017.712017

Received: November 4, 2017

Accepted: December 5, 2017

Published: December 8, 2017

Copyright (c) 2017 by authors and Scientific Research Publishing Inc. This work is licensed under the Creative Commons Attribution International License (CC BY 4.0).

http://creativecommons.org/licenses/by/4.0/

\begin{abstract}
A 46-year-old man was referred to our hospital due to severe ischemic mitral regurgitation with severe bileaflet tethering and a dilated left ventricle. We performed left ventricular reconstruction and mitral valve annuloplasty combined with papillary muscle relocation. Although left ventricular continued remodeling occurred during follow-up, left ventricular reconstruction and mitral valve annuloplasty combined with papillary muscle relocation provided durable mitral repair, and his functional status was good. Thus, this combined surgical treatment may reduce mitral regurgitation recurrence after mitral valve annuloplasty.
\end{abstract}

\section{Keywords}

Ischemic Mitral Regurgitation, Left Ventricular Reconstruction, Mitral Valve Annuloplasty, Papillary Muscle Relocation

\section{Introduction}

Mitral valve annuloplasty (MAP) has been the gold standard for surgical treatment of ischemic mitral regurgitation (IMR) [1]. However, high rates of persistence and/or recurrence of mitral regurgitation (MR) following MAP have been observed in patients with a dilated left ventricle (LV), and the surgical results of MAP have not been satisfactory. Recently, new and alternative surgical procedures that target the valvular, subvalvular, or ventricular levels have been at- 
tempted to provide longer-lasting MR relief, and promising results are reported by some groups [2] [3] [4] [5] [6]. Herein, we report a case involving LV reconstruction (LVR) and MAP combined with papillary muscle relocation (PMR) for severe IMR.

\section{Case Report}

A 46-year-old man was transferred to our hospital with a diagnosis of congestive heart failure with severe IMR. He had undergone percutaneous coronary intervention for the proximal left descending artery due to an acute myocardial infarction (MI) 6 months prior. A grade 2/6 pansystolic murmur was auscultated at the apex. His serum brain natriuretic peptide (BNP) level was $2598 \mathrm{pg} / \mathrm{ml}$, and his functional status was New York Heart Association (NYHA) class IV. Transthoracic echocardiogram (TTE) demonstrated severe IMR with an MR volume of $63 \mathrm{ml}$, effective MR orifice area of $0.7 \mathrm{~cm}^{2}$, tenting height of $1.5 \mathrm{~cm}$, tenting area of $3.0 \mathrm{~cm}^{2}$, anterior leaflet tethering angle (ALA) of $50^{\circ}$, posterior leaflet tethering angle (PLA) of $56^{\circ}$, and severe tricuspid regurgitation with pulmonary hypertension $(77 \mathrm{mmHg}$ ) (Table 1 ). Coronary angiography revealed no significant stenosis in any artery. Cardiac catheterization showed LV dilatation with akinesis at the mid-anterior wall to the apex, and fourth grade MR. The LV end-systolic volume index (LVESVI), LV ejection fraction (LVEF), pulmonary artery pressure, and pulmonary wedge pressure were $127 \mathrm{ml} / \mathrm{m}^{2}, 20 \%, 68 / 37$ $\mathrm{mmHg}$, and $43 \mathrm{mmHg}$, respectively. Transesophageal echocardiogram (TEE) showed severe central MR with tethering of both leaflets; in particular, the anterior leaflet was tethered due to secondary chordae and its mobility was severely restricted (Figure 1).

The LV was incised at the center of the scar in the anterior wall, and the exclusion line was determined based on a change in the color of the LV endocardium to white, as well as wall thinness. We placed 2-0 polytetrafluoroethylene (PTFE) sutures at the body of the anterior and posterior papillary muscles (PMs) thorough the LV incision. Horizontal aortotomy was performed and the sutures anchored to the PMs were passed from the LV cavity, through the aorta-mitral continuity, and underneath the commissure between the non-coronary and left coronary aortic cusps, and were exteriorized. The arteriotomy was closed and the PTFE sutures were tied with taut tension to relocate both PMs, while looking through the LV incision. The mitral valve was approached through a right-sided left atriotomy and MAP was performed using a $28-\mathrm{mm}$ semi-rigid full ring that was undersized by 1 size in relation to the intertrigonal distance. After the atriotomy was closed, the balloon in the LV cavity was filled $(100 \mathrm{~mL})$ to adjust the patch size, preventing excessive reduction in the LV volume. Then, the aortic cross-clamping was released and the left ventriculotomy was closed using an oval patch. Thereafter, intra-operative TEE demonstrated that the mobility of the anterior leaflet was remarkably improved, and MR had disappeared (Figure 2). 


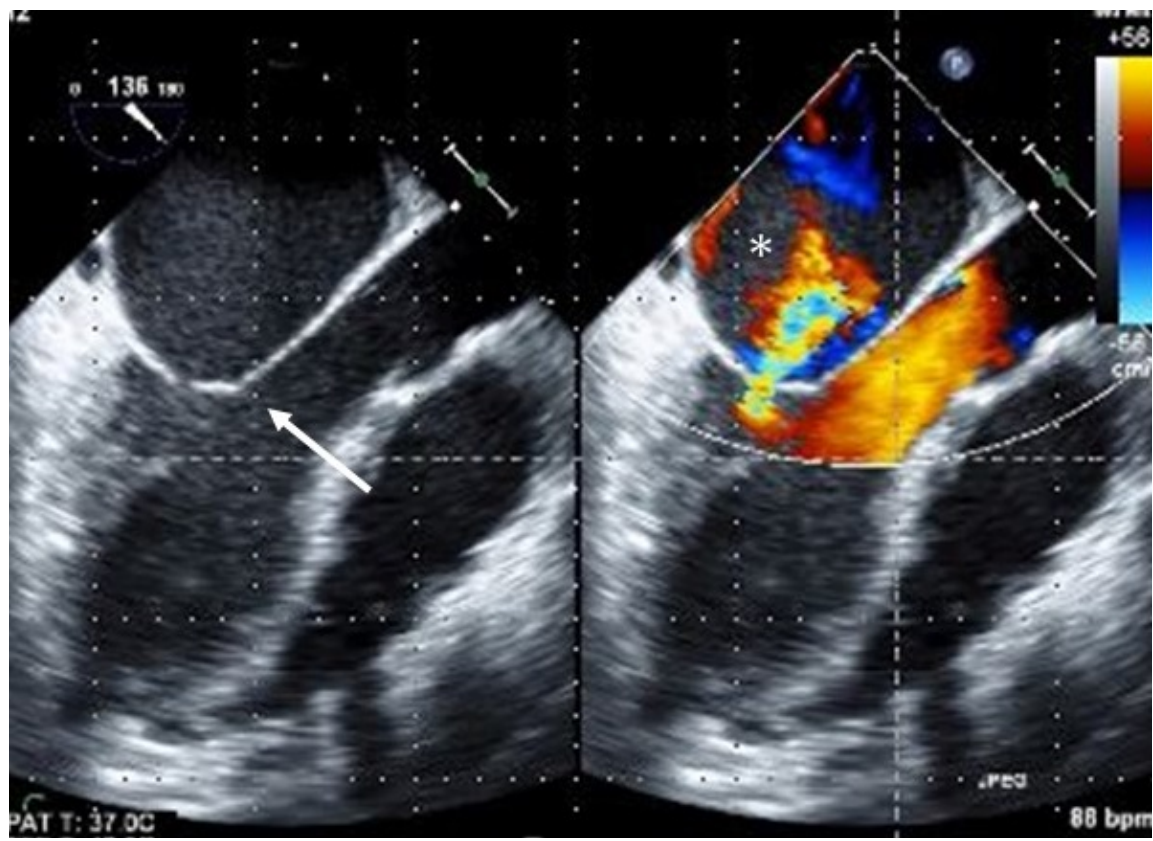

Figure 1. Preoperative transesophageal echocardiogram showing central mitral regurgitation (asterisk) with tethering of both leaflets; in particular, the anterior leaflet was tethered due to secondary chordae and its mobility was severely restricted (arrow).

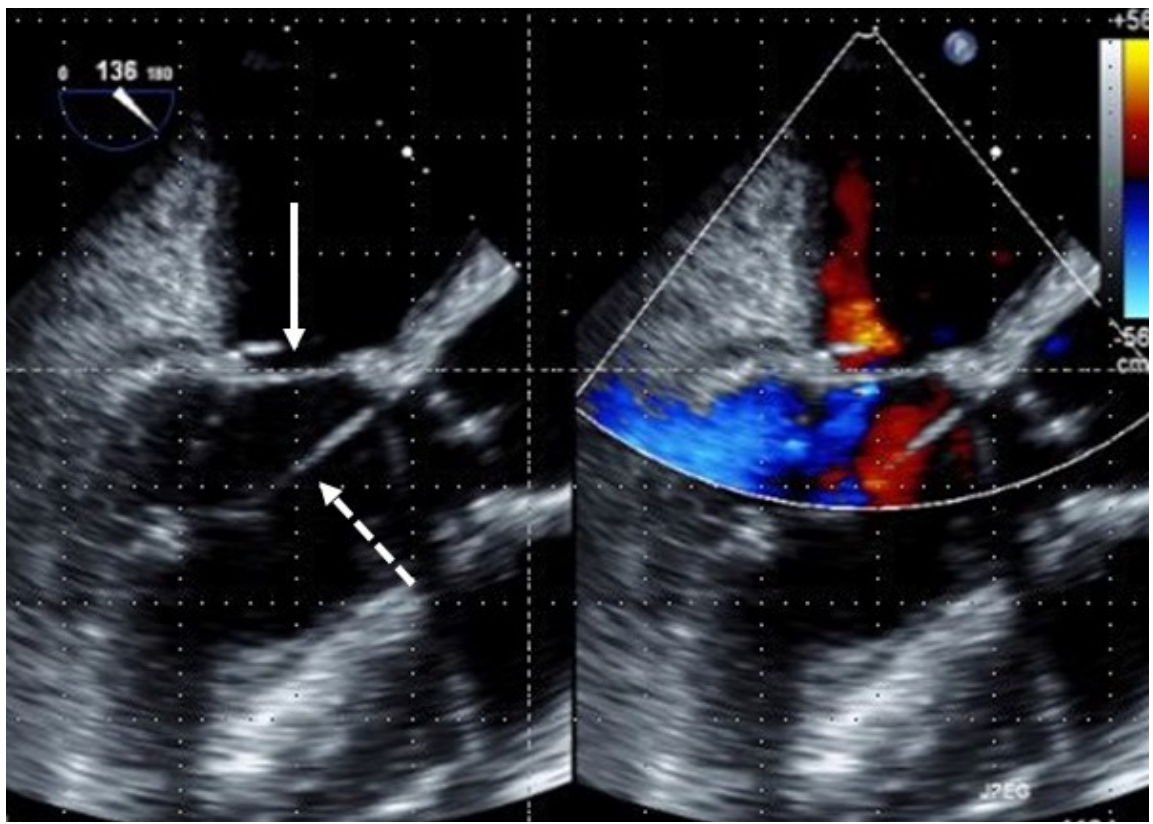

Figure 2. Intra-operative transesophageal echocardiogram showing mitral closure due to improvement in the mobility of the anterior leaflet (arrow) and no mitral regurgitation. Dashed arrow indicates relocating suture.

Post-operative cardiac catheterization showed that the LVESVI had decreased to $39 \mathrm{ml} / \mathrm{m}^{2}$, LVEF had increased to $50 \%$, and there was no MR. TTE demonstrated that the mitral valve worked as a functional monocusp valve with the anterior leaflet compensating for leaflet closure. Table 1 shows the pre- and post- 
Table 1. Pre- and post-operative transthoracic echocardiograms parameters.

\begin{tabular}{cccc}
\hline Parameter & Preoperative & Early postoperative At 32 months after operation \\
\hline LVDd (mm) & 60 & 54 & 66 \\
LVDs (mm) & 46 & 42 & 56 \\
LAD (mm) & 53 & 49 & 55 \\
Tenting height $(\mathrm{cm})$ & 1.5 & 0.2 & 0.7 \\
Tenting area $\left(\mathrm{cm}^{2}\right)$ & 3 & 0.3 & 1.2 \\
ALA (degree) & 50 & 0 & 18 \\
PLA (degree) & 56 & 86 & 76 \\
MR grade & severe & none & none \\
TR grade & severe & mild & mild \\
PAP (mmHg) & 77 & 42 & 31 \\
\hline
\end{tabular}

LVDd: left ventricular diastolic dimension; LVDs: left ventricular systolic dimension; LAD: left atrium dimension; ALA: anterior leaflet tethering angle; PLA: posterior leaflet tethering angle, MR mitral regurgitation, TR tricuspid regurgitation, PAP pulmonary artery systolic pressure.

operative TTE parameters. At 32 months after the surgery, the LV and left atrium had re-dilated. However, the PLA, which had deteriorated mildly in the early postoperative phase, did not worsen further and the ALA improved; recurrence of MR or pulmonary hypertension did not occur. His serum BNP level was $142 \mathrm{pg} / \mathrm{ml}$ and his functional status was NYHA class II.

\section{Discussion}

MAP has been the gold standard for surgical treatment of IMR [1]. However, high rates of persistence and/or recurrence of MR following MAP have been observed in patients with a dilated LV, because MAP may exaggerate posterior leaflet tethering and result in MR recurrence [7]. In general, symmetrical tethering after an anterior wall MI, which occurred in the present case, is more globally remodeled, spherical, and dysfunctional compared to asymmetrical tethering, which occurs mainly after an inferior wall MI; not only the posterior PM, but also the anterior PM, is displaced and the inter-papillary muscle distance increases. Consequently, the surgical results of MAP for symmetrical tethering are worse compared to those for asymmetrical tethering [8]. LVR for a dilated LV can improve mitral competence by reducing wall stress and decreasing the LV diameter. However, LV distortion may occur after LVR, exacerbating MR.

Because a suboptimal short axis/long axis ratio after LVR can influence the development of late MR, and LVR combined with a more physiological shape (surgical ventricular restoration) may be more beneficial for long-term results compared to that for a simple volume reduction surgery, we performed the septal anterior ventricular exclusion (SAVE) procedure [9]. However, MAP combined surgical ventricular restoration cannot always eliminate MR when the LV is remarkably remodeled; thus, an additional subvalvular procedure should be 
considered [6].

PMR corrects tethering at the subvalvular level, relocating an outwardly and apically displaced PMs toward the mitral annulus [3]. The posterior PM is usually a target for relocation in asymmetrical tethering [3] [4]; however, both PMs are targets in symmetrical tethering [5] [10]. Relocation toward the anterior annulus may allow natural directional repositioning of the PMs [4], effectively reducing the distance between the anterior annulus and the displaced PMs [4] and improving the diastolic anterior mitral leaflet excursion [10]. An approach through the aortotomy enables relocation of the PMs without disturbing mitral chordae tendineae, while precisely avoiding aortic regurgitation [4].

In addition to the exaggeration of posterior leaflet tethering [7], non-improvement in anterior leaflet tethering and continued LV remodeling are reportedly associated with persistence and/or recurrence of MR following MAP [8] [11]. In the present case, continued LV remodeling occurred despite LVR [12]. However, the posterior leaflet tethering never became exaggerated and the anterior leaflet tethering improved. Consequently, good MR control was maintained during the 32 months of follow-up. PMR for IMR is reportedly associated with good control of MR [4] [5], reversals in LV remodeling [4] [5], and fewer cardiac-related events [5]. However, PMR does not directly stabilize the posterior LV wall to reverse LV remodeling, and it is unknown whether the non-stabilized posterior LV wall influences the development of late MR after PMR. Moreover, long-term follow-up must be needed because our follow-up period may have been too short to determine the long-lasting efficacy of this combined surgical treatment for severe IMR.

\section{Conclusion}

We reported the surgical management of a patient with IMR, demonstrating that LVR and MAP combined with PMR is an effective procedure for severe bileaflet tethering and a dilated LV. Thus, this combined surgical treatment may reduce MR recurrence after MAP.

\section{References}

[1] Bolling, S.F., Deeb, G.M., Brunsting, L.A. and Bach, D.S. (1995) Early Outcome of Mitral Valve Reconstruction in Patients with End-Stage Cardiomyopathy. The Journal of Thoracic and Cardiovascular Surgery, 109, 676-683. https://doi.org/10.1016/S0022-5223(95)70348-9

[2] de Varennes, B., Chaturvedi, R., Sidhu, S., Côté, A.V., Shan, W.L., Goyer, C., et al. (2009) Initial Results of Posterior Leaflet Extension for Severe Type IIIb Ischemic Mitral Regurgitation. Circulation, 119, 2837-2843. https://doi.org/10.1161/CIRCULATIONAHA.108.831412

[3] Kron, I.L., Green, G.R. and Cope, J.T. (2002) Surgical Relocation of the Posterior Papillary Muscle in Chronic Ischemic Mitral Regurgitation. The Annals of Thoracic Surgery, 74, 600-601. https://doi.org/10.1016/S0003-4975(02)03749-9

[4] Langer, F., Kunihara, T., Hell, K., Schramm, R., Schmidt, K.I., Aicher, D., et al. (2009) RING+STRING: Successful Repair Technique for Ischemic Mitral Regurgi- 
tation with Severe Leaflet Tethering. Circulation, 120, S85-S91. https://doi.org/10.1161/CIRCULATIONAHA.108.840173

[5] Fattouch, K., Lancellotti, P., Castrovinci, S., Murana, G., Sampognaro, R., Corrado, E., et al. (2012) Papillary Muscle Relocation in Conjunction with Valve Annuloplasty improve Repair Results in Severe Ischemic Mitral Regurgitation. The Journal of Thoracic and Cardiovascular Surgery, 143, 1352-1355. https://doi.org/10.1016/j.jtcvs.2011.09.062

[6] Suma, H., Tanabe, H., Uejima, T., Isomura, T. and Horii, T. (2009) Surgical Ventricular Restoration Combined with Mitral Valve Procedure for Endstage Ischemic Cardiomyopathy. European Journal of Cardio-Thoracic Surgery, 36, 280-285. https://doi.org/10.1016/j.ejcts.2009.03.028

[7] Kuwahara, E., Otsuji, Y., Iguro, Y., Ueno, T., Zhu, F., Mizukami, N., et al. (2006) Mechanism of Recurrent/Persistent Ischemic/Functional Mitral Regurgitation in the Chronic Phase after Surgical Annuloplasty: Importance of Augmented Posterior Leaflet Tethering. Circulation, 114, I529-534. https://doi.org/10.1161/CIRCULATIONAHA.105.000729

[8] Gelsomino, S., Lorusso, R., Caciolli, S., Capecchi, I., Rostagno, C., Chioccioli, M., et al. (2008) Insights on Left Ventricular and Valvular Mechanisms of Recurrent Ischemic Mitral Regurgitation after Restrictive Annuloplasty and Coronary Artery Bypass Grafting. The Journal of Thoracic and Cardiovascular Surgery, 136, 507-518. https://doi.org/10.1016/j.jtcvs.2008.03.027

[9] Isomura, T., Horii, T., Suma, H. and Buckberg, G.D., RESTORE Group (2006) Septal Anterior Ventricular Exclusion Operation (Pacopexy) for Ischemic Dilated Cardiomyopathy: Treat Form Not Disease. European Journal of Cardio-Thoracic Surgery, 29, S245-S250. https://doi.org/10.1016/j.ejcts.2006.03.008

[10] Watanabe, T., Arai, H., Nagaoka, E., Oi, K., Hachimaru, T., Kuroki, H., et al. (2014) Influence of Procedural Differences on Mitral Valve Configuration after Surgical Repair for Functional Mitral Regurgitation: In Which Direction Should the Papillary Muscle Be Relocated? Journal of Cardiothoracic Surgery, 9, 185. https://doi.org/10.1186/s13019-014-0185-6

[11] Hung, J., Papakostas, L., Tahta, S.A., Hardy, B.G., Bollen, B.A., Duran, C.M., et al. (2004) Mechanism of Recurrent Ischemicmitral Regurgitation after Annuloplasty: Continued LV Remodeling as a Moving Target. Circulation, 110, II85-II90.

[12] Di Donato, M., Sabatier, M., Dor, V., Gensini, G.F., Toso, A., Maioli, M., et al. (2001) Effects of the Dor Procedure on Left Ventricular Dimension and Shape and Geometric Correlates of Mitral Regurgitation One Year after Surgery. The Journal of Thoracic and Cardiovascular Surgery, 121, 91-96.

https://doi.org/10.1067/mtc.2001.111379 